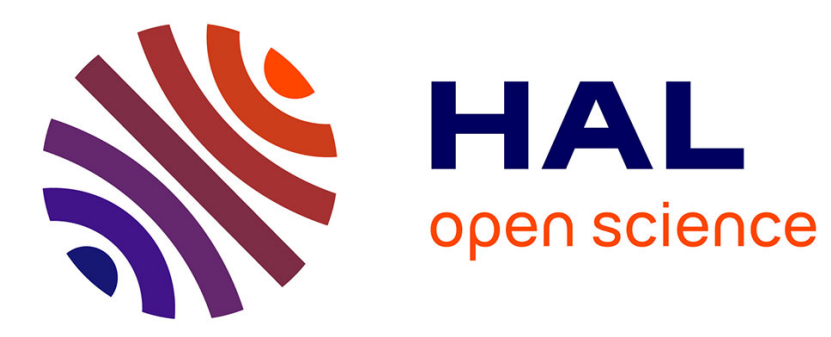

\title{
L'usager dans le système de santé : réformateur social ou fiction utile
}

\author{
Pierre Lascoumes
}

\section{To cite this version:}

Pierre Lascoumes. L'usager dans le système de santé: réformateur social ou fiction utile. Politiques et Management public, 2007, 25 (2), pp.129-144. 10.3406/pomap.2007.2371 . hal-01021649

\section{HAL Id: hal-01021649 \\ https://hal-sciencespo.archives-ouvertes.fr/hal-01021649}

Submitted on 9 Jul 2014

HAL is a multi-disciplinary open access archive for the deposit and dissemination of scientific research documents, whether they are published or not. The documents may come from teaching and research institutions in France or abroad, or from public or private research centers.
L'archive ouverte pluridisciplinaire HAL, est destinée au dépôt et à la diffusion de documents scientifiques de niveau recherche, publiés ou non, émanant des établissements d'enseignement et de recherche français ou étrangers, des laboratoires publics ou privés.

\section{(1) (1) $\$$}

Distributed under a Creative Commons Attribution - NonCommercial - NoDerivatives| 4.0 


\section{L'usager dans le système de santé : réformateur social ou fiction utile?}

In: Politiques et management public, vol. 25 n², 2007. pp. 129-144.

\section{Résumé}

En quelques années le « patient » s'est-il transformé en un « client » exigeant et procédurier qui déstabilise le rapport de confiance base de la relation médicale ? En donnant des pouvoirs à "l'usager " a-t-on déstabilisé le système de santé ou contribué à sa réforme par l'entrée en scène d'acteurs dotés de compétences originales ? L'article s'attache d'une part à différencier les formes de participation des usagers qui sont loin d'être homogènes. II montre ensuite comment des coopérations approfondies sont des facteurs de changement, mais aussi les limites de la participation observables de part et d'autre.

Citer ce document / Cite this document :

Lascoumes Pierre. L'usager dans le système de santé : réformateur social ou fiction utile ?. In: Politiques et management public, vol. $25 \mathrm{n}^{\circ} 2$, 2007. pp. 129-144.

http://www.persee.fr/web/revues/home/prescript/article/pomap_0758-1726_2007_num_25_2_2371 


\section{L'USAGER DANS LE SYSTEME DE SANTÉ : RÉFORMATEUR SOCIAL OU FICTION UTILE?}

\section{Pierre LASCOUMES*}

Fésumé

\begin{abstract}
En quelques années le "patient " s'est-il transformé en un "client " exigeant et procédurier qui déstabilise le rapport de confiance base de la relation médicale? En donnant des pouvoirs à "l'usager " a-t-on déstabilisé le système de santé ou contribué à sa réforme par l'entrée en scène d'acteurs dotés de compétences originales ? L'article s'attache d'une part à différencier les formes de participation des usagers qui sont loin d'être homogènes. II montre ensuite comment des coopérations approfondies sont des facteurs de changement, mais aussi les limites de la participation obsenables de part et d'autre.
\end{abstract}

* CEVIPOF-CNRS.

Revue POLITIQUES ET MANAGEMENT PUBLIC, Volume 15, n² 2, septembre 2007.

(C) Institut de Management Public - 2007. 
A écouter une grande partie des professionnels de la santé, médecins libéraux ou personnels de la santé, s'il y a un domaine où a été radicalement renversé le rapport de pouvoir entre une institution et ses usagers, c'est bien celui de la santé. En moins de dix ans, celui qui n'était qu'un patient (étymologiquement celui qui souffre et donc demande une aide extérieure à un professionnel) serait devenu un client exigeant, sûr et désinformé, contestataire, procédurier qui déstabiliserait l'indispensable rapport de confiance base de la relation médicale. Ceci renvoie à la question très générale de la répartition des pouvoirs dans les institutions; celles-ci ne peuvent-elles fonctionner que sur la base d'une forte dissymétrie? La reconnaissance de droits à un ancien dominé ne peut-elle déboucher que sur une nouvelle tyrannie?

En effet, du « client-centrisme » à la " tyrannie de l'usager » il n'y a qu'un pas. Ainsi, en novembre 2005, le Centre d'éthique médicale Maurice Rapin a organisé une journée d'étude ayant pour titre «Des patients toutpuissants ? ", consacrée au rôle montant des usagers et de leurs associations, ainsi qu'aux risques de judiciarisation croissante de la relation médicale ${ }^{1}$. Un autre exemple récent nous est fourni par la campagne lancée en septembre 2005 par la puissante Fédération Hospitalière de France (FHF) qui a mené une opération médiatique nationale (affichage, spot TV, publication presse généraliste et professionnelle) au sujet des « incivilités à l'hôpital ». " Incivilité », le terme est explicite et emprunte directement aux programmes de lutte contre la criminalité en incitant à réagir aux transgressions de faible gravité (tags, casse de vitres et saccage de cabines téléphoniques) afin d'affirmer une "tolérance zéro " aux actes dits " incivils" perturbant la vie collective. Regardons de quoi est faite cette hostilité professionnelle au "client-centrisme". Trois visuels montrent des professionnels occupés dans leur tâche (surveillance d'un écran, oscultation, examen d'une radio), chaque image est accompagnée d'une phrase ironique (respectivement) : "Si on ne vous parle pas, c'est qu'on regarde la télé ", "Si vous patientez, c'est que d'autres se font masser ", "Si vous attendez, c'est qu'on préfère regarder des photos "). Chaque fois, l'usager est assimilé à un sujet égocentrique, aussi impatient que stupide. II ne comprend pas le contexte organisationnel où il se trouve et s'insurge violemment. En bas de page figure la phrase "On fait notre maximum, restez poli, au minimum », suivie de la référence à deux articles du code pénal qui incriminent les agressions physiques et verbales. C'est là une façon bien unilatérale de poser le problème récurrent des attentes à l'hôpital, des difficultés particulières à les gérer et à informer dans un contexte de pénurie de personnels, mais aussi dans un cadre organisationnel où le temps du médecin est un bien absolu et où celui du patient n'existe pas.

\footnotetext{
${ }^{1}$ Devant les TGI, il y a eu 5000 demandes en 2003 (référés + fond) qu'il faut ramener aux 500 millions d'actes médicaux annuels. En outre, il y a environ 4000 demandes devant les $\mathrm{CRCl}$ (commissions d'indemnisation des accidents médicaux créées en 2002).
} 

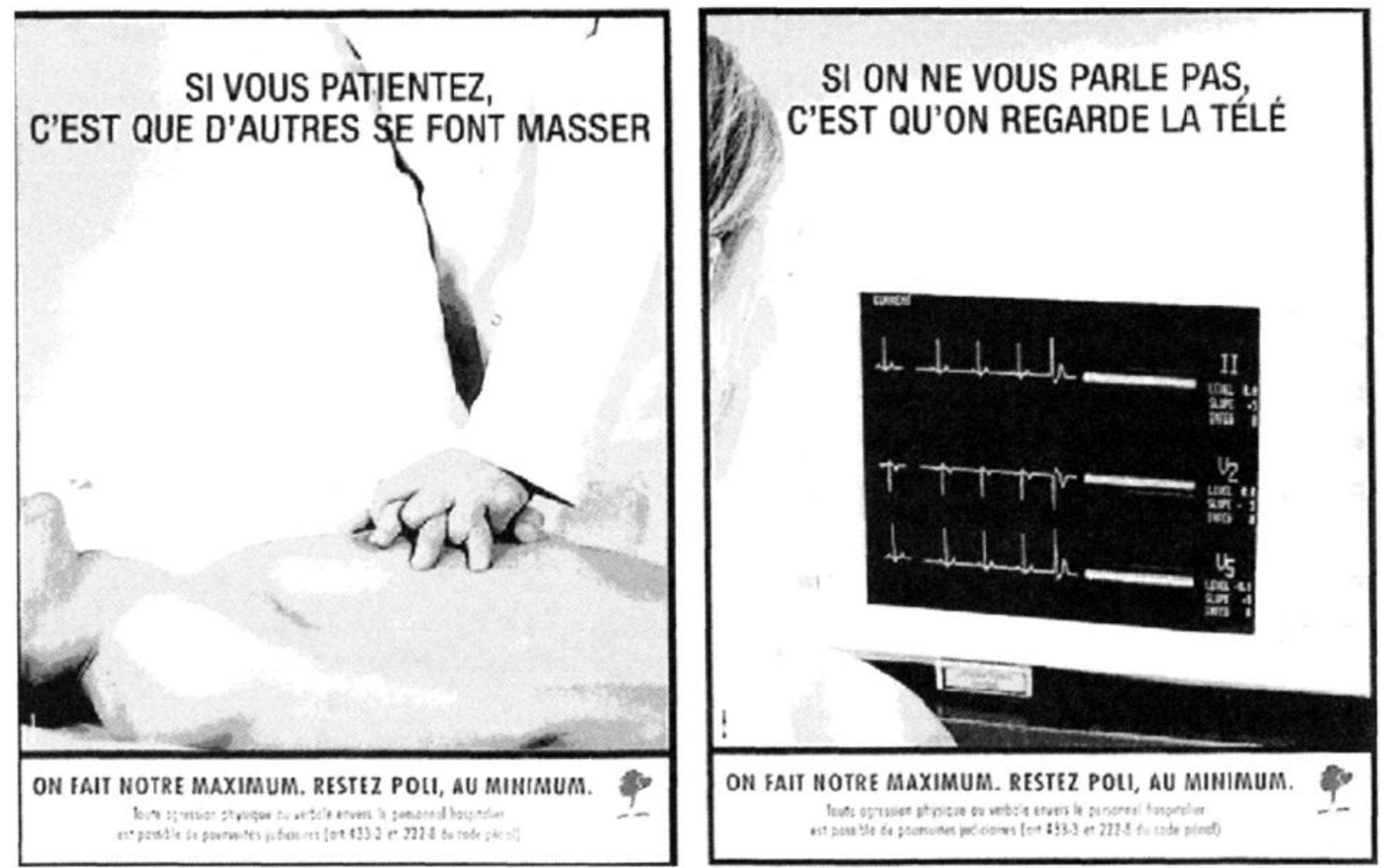

La référence au " client-centrisme " est sous-tendue par deux postulats. Tout d'abord, l'idée selon laquelle les références au "client ", c'est-à-dire à "l'usager consumériste», auraient pris une telle place qu'elles seraient devenues les concepts centraux des programmes d'action des services publics. Ensuite, l'expression de "client-centrisme » a une connotation négative. Elle laisse entendre que la prise de parole des usagers sur la conception du service public n'aurait eu aucun impact positif (sur la qualité, le prix des prestations, etc.), seulement des impact négatifs. Enfin, ce phénomène s'opposerait à la citoyenneté dans la mesure où il substituerait la revendication individualiste à la défense de l'intérêt collectif (celui de tous les usagers), voire de l'intérêt général. D'une part, cette problématique me semble reprendre sans beaucoup de distance critique le point de vue des acteurs professionnels. Ce n'est pas parce que beaucoup d'entre eux sont dominés par la défense des routines corporatistes et les catégories analytiques du management qu'il faut s'en tenir là pour organiser la réflexion sur les changements intervenus depuis une vingtaine d'année dans les interactions entre les organisations publiques et leurs usagers. D'autre part, il me semble que les réflexions contemporaines permettent de différencier trois types de destinataires: l'administré, l'usager et le client; et, partant de là, trois types de relation ${ }^{1}$. La notion d'administré renvoie à un destinataire passif de la règle publique, on attend de lui une conformité, une soumission disciplinée. La notion d'usager est liée à une conception du service public qui donne des droits et des devoirs, et mieux des responsabilités, à ceux qui

\footnotetext{
1 Michel Chauvière, Jacques T. Godbou, Les usagers entre marché et citoyenneté, L'Harmattan, 1992Philippe Warin, Quelle modernisation des senvices publics? Les usagers au cœur de la réforme, Paris, La Découverte, 1997 - Jean-Marc Weller, « Usagers comme ils disent », Vacarmes, n 12, 2000, p. 12 s. et L'Etat au guichet, Desclée de Brower, 1999 - Vincent Dubois, La vie au guichet, relation administrative et traitement de la misère, Economica, 2003.
} 
bénéficient des prestations organisées par la collectivité. Enfin, la notion de client renvoie à une relation contractuelle individualisée dont la dimension économique est centrale.

Comment penser alors ces nouveaux rapports entre professionnels et destinataires ? Certes la répartition des pouvoirs entre administration et administré a été fortement interrogée durant les deux dernières décennies et un certain nombre de réponses ont été expérimentées pour dépasser le clivage fonctionnel et politique qui les opposait. Dans ce sens différentes formes de participation ont été introduites, mais jusqu'où sont-elles parvenues à faire évoluer les relations hiérarchiques antérieures '? Pour le dire autrement, même dans un contexte de "client-centrisme » apparent, jusqu'à quel point «le client est-il roi »? Pour répondre à ces questions je m'appuierai sur une distinction des formes de la démocratie participative proposée par M.H. Bacquet, H. Rey, Y. Sintomer ${ }^{2}$ et mise à l'épreuve au domaine de la santé par $P$. Hassenteufel. Ils distinguent cinq niveaux de participation: le premier est celui du discours managérial qui conçoit le rapport à la clientèle comme une technique de réorganisation et de mobilisation du personnel; le second correspond à des efforts de modernisation des fonctionnements organisationnels par le renforcement de la communication avec les usagers, les dispositifs d'information (Charte Marianne ${ }^{3}$ ), les structures d'accueil, le recueil des opinions (enquête de satisfaction); le troisième correspond aux formes de démocratie de proximité qui s'efforcent de structurer dans une certaine continuité les échanges sur la connaissance des besoins, la recherche de solutions et le suivi des décisions prises; le quatrième niveau est celui de "l'empowerment " (terme difficilement traduisible) qui renvoie à la reconnaissance d'une identité légitime et à l'attribution de moyens d'action (juridiques et financiers) à des groupes de contre-pouvoir; le cinquième est le niveau d'une démocratieparticipative pleine et entière qui combine différents types de représentation (politique, groupes d'intérêts organisés, minorités) et articule des procédures de délibération différenciées.

II me semble que l'on peut sur cette base s'interroger sur le degré d'ouverture effectué par les organisations à l'égard de leurs destinataires. Je prendrai l'exemple des politiques de santé qui ont connu en quelques années des évolutions significatives. Mais jusqu'où sont-elles réellement allées? Comment se situent les innovations introduites par rapport aux cinq niveaux de participation présentés ci-dessus? Depuis une vingtaine d'années le pouvoir médical basé sur un mélange de paternalisme et de savoir scientifique qui dominait l'ensemble du système médical (du face à face libéral à la santé publique) a connu beaucoup de transformations ${ }^{4}$. Le temps

\footnotetext{
Yves Sintomer, Le pouvoir au peuple, jurys citoyens, tirage au sort et démocratie participative, La Découverte, 2007.

2 Marie-Hélène Bacqué, Henri Rey, Yves Sintomer, «La démocratie participative urbaine face au néolibéralisme ", Mouvements, 39, mai-juin 2005- Yves Sintomer, Le pouvoir au peuple, jurys citoyens, tirage au sort et démocratie participative, La Découverte, 2007.

${ }^{3}$ Le 2 mars 2004 a été publiée par le secrétaire d'Etat à la réforme de l'Etat « la Charte Marianne " qui est une charte de qualité de service qui s'impose à toutes les administrations accueillant du public. Un «baromètre de la qualité des services » devrait permettre d'assurer le suivi de cette mesure.

4 Patrick Hassenteufel, "Vers le déclin du pouvoir médical ? Un éclairage européen, France, Allemagne ", Pouvoirs, N89, 1999.
} 
où le patient s'en remettait au savoir-faire unilatéral de celui qui peut le conseiller, le soigner, organiser sa prise en charge, ce temps est-il vraiment dépassé ? Ce nouveau venu, l'usager (celui qui est dans un rapport contractuel de droit privé ou public) est-il pour autant un acteur consistant, capable d'exercer un pouvoir autonome? L'unanimisme actuel mérite quelques interrogations sur la représentation des usagers et sur les pouvoirs de ces porte-paroles. La référence systématique à " l'usager ", ses savoirs et ses besoins n'est-elle qu'un nouveau gadget bureaucratique renouvelant la légitimité de pratiques fondamentalement inchangées? Ou bien, ce personnage obligé atteste-t-il la constitution d'un nouveau type d'acteur capable d'analyses et d'actions libérées de l'emprise de l'institution médicale et sujet d'une parole libre ? Pour discuter et relativiser l'hypothèse du « clientcentrisme » en matière de santé, on peut facilement opposer le point de vue des professionnels et celui des associatifs :

\begin{tabular}{|c|c|c|}
\hline $\begin{array}{l}\text { Degré d'importance de la } \\
\text { place de l'usager selon }\end{array}$ & $\begin{array}{l}\text { Le point de vue des } \\
\text { professionnels }\end{array}$ & $\begin{array}{l}\text { Le point de vue des } \\
\text { associatifs }\end{array}$ \\
\hline 1 - niveau managérial & $\begin{array}{l}\text { Fort - l'usager est au centre } \\
\text { du système de soin » }\end{array}$ & $\begin{array}{l}\text { Faible } \quad \text { les logiques } \\
\text { d'organisation professionnelle } \\
\text { sont toujours prédominantes }\end{array}$ \\
\hline 2 - niveau de la modernisation & $\begin{array}{l}\text { Fort - L'information du patient } \\
\text { et la qualité de son accueil sont } \\
\text { centraux }\end{array}$ & $\begin{array}{ll}\text { Faible - plus d'actions } & \text { de } \\
\text { communication que } & \text { de } \\
\text { changements substantiels } & \end{array}$ \\
\hline $\begin{array}{l}3 \text { - niveau de démocratie de } \\
\text { proximité }\end{array}$ & $\begin{array}{l}\text { Moyen - Actions d'ouverture } \\
\text { sur l'environnement social, } \\
\text { comités d'usagers }\end{array}$ & $\begin{array}{l}\text { Faible - participation formelle à } \\
\text { de nombreuses structures, } \\
\text { l'usager est plus souvent une } \\
\text { caution qu'un acteur }\end{array}$ \\
\hline 4 - niveau de l'empowerment & $\begin{array}{l}\text { Fort - les associations sont } \\
\text { financées, disposent de } \\
\text { moyens " maison d'usager », } \\
\text { consultées sur tout }\end{array}$ & $\begin{array}{l}\text { Moyen - pouvoirs conquis mais } \\
\text { fragiles. } \\
\text { Financement, agrément, auto- } \\
\text { organisation (CISS) }\end{array}$ \\
\hline $\begin{array}{l}5 \text { - niveau de la démocratie } \\
\text { participative }\end{array}$ & $\begin{array}{l}\text { Faible - une dynamique en } \\
\text { cours }\end{array}$ & $\begin{array}{l}\text { Faible - démocratisation en } \\
\text { grande partie formelle, force du } \\
\text { backlash médical }\end{array}$ \\
\hline
\end{tabular}

Le point de vue sociologique doit se tenir à égale distance de l'une et de l'autre de ces conceptions. C'est pourquoi je dois préciser d'entrée que ma position est un peu particulière dans la mesure où je suis en quelque sorte "un acteur concerné ». J'ai en effet participé (à ma mesure) aux changements analysés ici en tant qu'acteur associatif, ayant été successivement volontaire puis responsable mandaté d'une association de personnes malades (Aides), puis en tant qu'animateur d'un collectif associatif (CISS Collectif interassociatif sur la santé). Je n'ai plus aujourd'hui de mandats et je m'efforce de penser les processus dans lesquels nous avons été engagés. Mon point de vue doit donc être considéré comme celui d'un " témoignage d'acteur » avec les fragilités qui y sont, bien évidemment, liées.

Je commencerai par rappeler les grandes étapes de la reconnaissance de l'usager du système de santé comme acteur autonome, puis je présenterai ce qui me semble être aujourd'hui les points forts et les points faibles de cette mobilisation, avant de conclure sur les possibilités et les ambiguïtés d'un tel 
contre-pouvoir. Tout ceci pour étayer l'hypothèse gradualiste de $P$. Hassenteufel selon laquelle la reconnaissance par une organisation de l'intérêt d'une ouverture participative ne signifie nullement un retournement pur et simple de pouvoir. Quant à parler ici de « client-centrisme » ...

L'émergence de nouveaux acteurs
Depuis l'ordonnance Juppé de 1996 sur l'organisation hospitalière, en surface que de changements ! II n'est plus un propos de professionnel de la santé, ni de responsable de la politique en ce domaine qui ne prétende que "l'usager doit être au centre du système de santé ». Pas un colloque, pas un groupe de travail sans qu'on ne veuille y faire figurer un « représentant des usagers ou des malades ". Voilà les représentants des usagers en proximité des problèmes dans les conseils d'administration des hôpitaux, dans les commissions régionales de conciliation et d'indemnisation, mais aussi dans des organismes expert comme la Haute Autorité de Santé (HAS), I'Institut National de Veille Sanitaire (INVS) ou le conseil de surveillance de la CNAM. La loi sur « les droits des malades et la modernisation du système de santé " du 4 mars 2002, celle sur la santé publique du 9 août 2004 témoignent de la prise en compte par le politique de ce nouvel acteur.

Les prises de parole contemporaines des usagers de la santé, leur progressive prise d'autonomie et l'acquisition de pouvoirs reconnus trouvent une de leurs principales origines dans le mouvement des Alcooliques anonymes aux USA dans les années cinquante, puis dans le mouvement des communautés thérapeutiques d'usagers de drogues dans les années soixante-dix (Synanon, Daytop) ${ }^{1}$. Ces groupements de personnes désireuses de rompre avec une dépendance se donnèrent des principes radicaux qui introduisaient une rupture décisive en leur permettant de se déprendre des discours médicaux et juridiques qui les objectivaient depuis des décennies et assuraient une continuité entre une aliénation et l'autre. La faiblesse des réponses médicales et l'importance des problèmes psycho-sociaux auxquels ils étaient confrontés conduisirent de petites minorités à s'auto-organiser. Ils transformèrent les groupes de parole à visée thérapeutique en "groupe d'auto support ${ }^{2}$. Chacun pouvait y trouver un cadre pour tenter de s'y reconstruire, selon la logique de son expérience personnelle et en s'appuyant sur un support collectif.

Ce modèle a servi de référence, plus ou moins explicite, aux mobilisations sociales qui sont apparues à l'occasion de l'épidémie du VIH, puis lors de la prise de conscience collective de l'abandon des maladies génétiques transmissibles (myopathie, mucoviscidose). Les points communs avec les situations précédentes étaient nombreuses : absence de réponse médicale, existence d'un rejet social, entremêlement de facteurs sanitaires, psychologiques et individuels, capacité d'auto-organisation. Dans tous ces cas l'action collective a servi de base à des changements importants à trois niveaux : la capacité à assumer individuellement une maladie, l'évolution des

\footnotetext{
1 Jacques Durand-Dassier, Psychothérapies sans psychothérapeutes, Editions de l'Epi, Paris, 1970.

${ }^{2}$ Groupes basés sur les principes de libre expression, d'empathie et de non-jugement.
} 
relations malade/médecin, et la politisation du problème ${ }^{1}$. Ces transformations peuvent être précisées de deux façons. D'une part, l'organisation, via des associations spécifiques, de la prise de parole des personnes atteintes s'est traduite par la constitution de collectifs capables de développer aussi bien des pratiques d'auto-support et de défense des droits, que de structurer des groupes d'action publique ayant une capacité d'expression originale. D'autre part, cette mobilisation s'est accompagnée de la constitution d'une compétence profane, susceptible de produire des savoirs spécifiques, d'interpeller les discours et les pratiques des professionnels, et de participer à la définition des démarches cliniques de soin et de santé. Ces changements touchent plus largement les relations entre les usagers du système de santé et les institutions publiques et privées ${ }^{2}$. Ces groupes ont retiré des apports significatifs des transformations contemporaines de la recherche biomédicale. Depuis le Code de Nuremberg (1948) de nombreux textes internationaux ont régi les rapports médecin/malade dans le cadre des expérimentations afin de garantir aux patients que rien ne serait réalisé sur eux sans leur pleine information et sans l'obtention d'un consentement éclairé $^{3}$. Mais ces énoncés étaient restés assez formels jusqu'à la mobilisation particulière des personnes atteintes par le VIH. Aux Etats-Unis comme en France, des travaux approfondis ont été accomplis sur les dispositifs de suivi permanent des protocoles de recherche $e^{4}$ et sur le vaste répertoire d'interactions développé entre cliniciens et représentants des malades ${ }^{5}$. Les contributions de ces collectifs mêlant professionnels et nonprofessionnels dépassent largement les seules questions de la qualité de l'information et la vigilance sur les conditions de recueil du consentement telles qu'elles sont cadrées par la loi du 20 décembre 1988 concernant la protection des personnes participant aux recherches bio-médicales et qui sont garanties, en théorie, par les Comités consultatifs de protection des personnes. L'idée fondamentale est que la totalité des éléments du dispositif de recherche et de traitement doit être prise en considération par tous et surtout doit rester discutable durant la totalité du processus, des données chimiques et biologiques jusqu'aux éléments de qualité de vie, en passant par les rythmes d'examen et les procédures de validation.

Fin 1998, reprenant le modèle des états généraux des personnes atteintes du sida de $1990^{6}$, ont eu lieu, à trois mois d'intervalle, des états généraux de personnes atteintes du diabète, et de celles atteintes du cancer ${ }^{7}$. Cette approche centrée sur la prise de parole des personnes atteintes rompt explicitement avec les démarches classiques des états généraux "du

\footnotetext{
«Politisation », au sens de mise en débat public, de mobilisations d'acteurs et de définition d'action collectives publiques et privées - Steve Epstein, La grande révolte des malades (histoire du sida), (1996), Paris, Les empêcheurs de penser en rond, 2001: Nicolas Dodier, Leçons politiques de l'épidémie de sida, Paris Editions EHESS, 2003 ; Christophe Brocca, Ch., Agir pour ne pas mourir, Act Up, les homosexuels et le sida, Presses de Sciences-Po, 2005.

${ }^{2}$ Michel Callon, Pierre Lascoumes, Yannick Barthe, "A spécialiste, spécialiste et demi ", chap. 3, Agir dans un monde incertain, Le Seuil, Paris, 2001, p. 105 s.

${ }^{3}$ Noëlle Lenoir, Bertrand Mathieu, Les normes internationales de la bioéthique, PUF, 1998.

${ }^{4}$ Par exemple en France le groupe TRT5 de l'Agence nationale de recherche sur le sida (ANRS).

5 Jeanine Barbot, Les malades en mouvements, la médecine et la science à l'épreuve du sida, Balland, 2002 Sébastien Dalgalarondo, Sida : la course aux mollécules, Paris Editions EHESS, 2004.

${ }^{6}$ Vivre le sida, Le livre blanc des états généraux, Cerf, Paris, 1992.

${ }^{7}$ Qui ont été renouvelés en novembre 2000
} 
diabète" ou "du cancer" dominées jusque là par les points de vue professionnels. Ces prises de parole étaient souvent émotionnelles, parfois violentes, mais toujours révélatrices de vécus particuliers de la maladie et de l'extrême diversité des pratiques médicales ${ }^{1}$. Ces énoncès témoignaient de la volonté croissante des personnes directement concernées d'exprimer des points de vue et de les faire reconnaître comme légitimes, d'entrer en discussion avec les professionnels et de revendiquer des positions spécifiques. Un autre exemple est donné par le rôle croissant de l'association française des myopathes dans le développement de stratégies de recherche qui associent les personnes atteintes aux généticiens et biologistes de très haut niveau, et cela grâce aux ressources du téléthon ${ }^{2}$. Enfin, au printemps 1999 se sont déroulés des Etats généraux de la santé. Malgré le caractère hètérogène de leurs résultats ${ }^{3}$, ils ont amorcé une dynamique qui a débouché sur la loi du 4 mars 2002 qui articule trois dimensions essentielles: le renforcement des droits individuels (information, consentement, accès direct aux informations médicales, non-discrimination, etc.), celui des droits collectifs (agrément des associations, statut des représentants, création d'une instance de conciliation régionale, participation aux conférences régionales, etc.), et deux procédures, l'une de conciliation en cas de conflit, l'autre d'indemnisation des victimes d'aléas thérapeutiques.

Un autre phénomène important de ces dernières années réside dans la constitution de nouveaux groupes cherchant à organiser une prise de parole collective et à formuler des revendications communes face aux institutions de santé. C'est pour assurer le suivi de la réforme hospitalière de 1996 qu'a èté créé (en juin de cette année là) par une douzaine d'associations le Collectif interassociatif sur la santé (CISS) ${ }^{4}$. Dix ans plus tard il regroupe vingt-six des principales associations nationales de personnes malades, de personnes handicapées, de consommateurs et des associations familiales ${ }^{5}$, il dispose ainsi d'une représentativité nationale. Cette démarche s'est concrétisée par un ensemble d'actions ${ }^{6}$. Les principaux dossiers de fonds concernent tout d'abord les travaux sur l'accès des personnes aux informations relatives à leur santé : conditions d'accès direct à l'ensemble des documents, suivi des travaux du Conseil supérieur des systèmes d'information de santé, et de ceux des groupes de travail préparant la carte Sésame-Vital. S'y rattache également le travail de réflexion critique sur le fonctionnement des commissions de conciliation et les besoins en matière de réforme de l'expertise médicale et d'indemnisation des accidents médicaux. S'inscrit

\footnotetext{
${ }^{1}$ La Ligue contre le cancer, Les malades prennent la parole, Ramsay, Paris, 1999.

${ }^{2}$ Vololona Rabeharisoa, Michel Callon, Le pouvoir des malades, I'AFM et la recherche, Presses de l'école des mines, Paris, 1999.

${ }^{3}$ In Callon et al., op cit, p. 248-249.

${ }^{4}$ Le CISS s'est constitué à la suite des travaux de la commission Devulder (1995) qui préparait les ordonnances Juppé de 1996. Un représentant associatif en était membre et organisa une audition collective des principales associations de santé. Ce fut un moment de cristallisation décisif. L'UNAF y présenta les résultats d'une enquête d'ampleur nationale sur les conditions d'hospitalisation vécues par les usagers.

${ }^{5}$ Le CISS regroupe dans un collectif (organisé en association depuis 2005) les associations suivantes : AFD - AFH - AFLM - AFM - AFP - AIDES - Alliance maladies rares - APF - CSF - FFAAIR - Familles Rurales FNAMOC - FNAPSY - FNATH - La ligue contre le cancer - Le Lien - ORGECO - RESHUS - UFCS - UNAF UNAFAM - UNAPEI - VLM. LU UFC, Que Choisir? qui était membre fondateur s'est distancié en 2005 pour des raisons de principe lorsque le CISS s'est constitué en association 1901.

${ }^{6}$ Tous les documents produits par le CISS sont accessibles sur le site : www.leciss.org
} 
aussi dans cette perspective la participation aux travaux de l'ANAES (aujourd'hui HAS), à son conseil d'administration, à son conseil scientifique de l'évaluation et de l'accréditation (aujourd'hui certification), et à différents groupes de travail ayant préparé les référentiels. On peut y adjoindre d'autres dossiers de fonds, très techniques, comme par exemple celui portant sur l'accès à l'assurance de tous les risques aggravés (différenciation des besoins d'assurance, clarification de la hiérarchie des risques aggravés, détermination des surprimes) et aujourd'hui aux travaux consacrés à la réforme du financement de l'assurance maladie qui ont permis aux représentants d'usagers d'accéder aux instances nationales et départementales en ce domaine ${ }^{1}$.

\section{Points forts et point faibles d'une mobilisation}

Si l'on essaye maintenant de positionner les acquis de cette mobilisation par rapport aux cinq types (ou degré) de participation présentés en introduction, les résultats sont pour le moins balancés. Mais, globalement, l'observation empirique des pratiques semble plutôt contredire l'hypothèse du « client-centrisme ". Les changements observés peuvent être classés du niveau quatre l'empowerment au niveau un " managérial ". Certes, s'il n'y avait pas eu une revendication forte et organisée des représentants d'usager, il n'y aurait pas eu de réforme de l'accès au dossier médical ou de mise en place d'un système d'indemnisation des aléas médicaux. Mais, dans beaucoup de domaines, la prise en compte des demandes reste beaucoup plus parcellaire, dépendante des contextes organisationnels locaux où dominent toujours les logiques professionnelles classiques.

\section{Empowerment et engagement dans la démocratie de proximité : une mobilisation citoyenne des usagers}

Deux dimensions permettent d'argumenter dans le sens de l'existence de changements approfondis. Ils donnent un sens concret aux termes d'empowerment et "engagement dans la démocratie de proximitè ", ils contribuent ainsi à une mobilisation citoyenne qui enrichit globalement le bien commun et fait bénéficier tout un chacun du gain de démarches initialement spécifiques :

- du prescriptif au coopératif : tout d'abord les associations d'usagers de la santé ont permis une autonomisation d'un point de vue et d'une parole d'usager dans un milieu professionnel qui était jusqu'au milieu des années quatre-vingt largement dominé par une culture paternaliste et des croyances scientistes. Les crises sanitaires, et au premier chef celle liée au sida, ont fourni aux personnes malades l'occasion de se déprendre du discours médical qui les objectivait unilatéralement.

\footnotetext{
${ }^{1}$ Loi du 13 août 2004 sur la réforme de l'assurance maladie.
} 
Le modèle classique de la clinique repose sur l'imposition d'un regard médical, épuré de toute contingence humaine et sociale' ${ }^{\prime}$ II n'accorde aucune place au profane qui ne peut être qu'un d'objet d'examen et d'intervention. Ce modèle prescriptif a parfois évolué vers un modèle supplétif lorsque les professionnels ont pris conscience des différentes limites de l'approche strictement scientifique de la santé qui raisonne à un haut niveau d'abstraction et en déréalisant la situation de maladie. Malgré sa forte technicisation depuis cinquante ans, le savoir médical s'est révélé marqué d'incertitudes difficilement cadrables (complexité des symptômes, limites des traitements, existence d'effets non-désirés -maladies iatrogènes- infections nosocomiales); et des failles dans les organisations médicales sont apparues (traitement de l'urgence, mauvaise coordination de soins, perversion du système par les logiques économiques). Tous ces facteurs perturbateurs ont progressivement érodé la croyance, celle des profanes comme celle des professionnels, dans la toute puissance et le caractère strictement bienfaiteur de la médecine. Une bonne connaissance scientifico-technique est insuffisante à elle seule pour garantir le soin.

De plus, chaque pathologie est incarnée dans un vécu individuel et dans un certain degré de complexité organique et biologique. La médecine n'a pas affaire à un public homogène et constant. Le médecin est confronté à des personnes différenciées selon des facteurs socio-culturels et des modes de vie. Ainsi, chaque sujet est dépositaire d'une expérience, d'un savoir spécifique qui est le fruit de l'observation individuelle, du rapport de soi à soi et qui enrichit le savoir abstrait du spécialiste à condition de pouvoir être exprimé et entendu. Ces savoirs pratiques viennent compléter et épauler les savoirs scientifiques et cliniques classiques. Ce modèle supplétif a pris une importance croissante dans les situations où il y a incertitude scientifique sur les diagnostics (maladies génétiques rares) ou sur les traitements (cancers, sida). Plus la situation est controversée, moins il existe de réponses validées et généralisables, plus il est nécessaire d'élargir l'espace de discussion, de donner la parole à des acteurs et des points de vue diversifiés.

Dans ce modèle supplétif, les situations de crise ne proviennent plus des pertes de confiance, mais de la violence des prises de paroles. Soudain émergent dans l'espace public des mots, des cris, des accusations, des revendications jusque là tues et qui jaillissent avec la force de ce qui a été longtemps refoulé. Confrontés à des mises en cause qui interpellent violemment un pouvoir technico-paternaliste, beaucoup de professionnels et d'institutions sont déstabilisés. En effet dans le modèle supplétif, la prise de parole doit rester cantonnée dans la relation asymétrique. Elle n'est recevable que comme force d'appoint. Le profane doit rester un palliatif du professionnel, il ne peut prétendre à l'égalité.

La période qui est amorcée depuis le milieu des années 80 essaye d'introduire une plus grande symétrie du savoir et du pouvoir dans un modèle coopératif entre professionnel et usager. Ce rééquilibrage passe par la reconnaissance du patient comme sujet et de l'usager comme interlocuteur. La prise de parole du profane prend ici une toute autre valeur, elle n'est plus

\footnotetext{
${ }^{1} \mathrm{M}$. Foucault a bien décrit « le mythe du libre regard qui dans sa fidélité à découvrir, reçoit la vertu de détruire ; libéré de l'ombre, il dissipe les ombres ", Naissance de la clinique, PUF, 1963, p. 51.
} 
tolérée ou produite par des crises, elle correspond à une recherche d'égalisation des échanges entre professionnel et non-professionnel. Elle est sous-tendue par l'affirmation d'un savoir et d'un pouvoir propre au profane concerné. Ce modèle repose sur deux principes fondamentaux. Tout d'abord, celui de la reconnaissance de deux compétences, différentes certes, mais chacune ayant une légitimité particulière. Le savoir médical général et abstrait doit entrer en dialogue avec le savoir sur soi, individuel et incarné, de la personne concernée. Ensuite, chacun des acteurs engagés dans la relation doit disposer de droits équivalents à l'expression de ses besoins, de ses buts et à la participation à la décision. Les différents savoirs visent à devenir indissociables. Une coopération symétrique est au prix de leur reformulation et de leur coordination. Chaque savoir doit pouvoir être développé et transmis ; l'un et l'autre doit pouvoir être ajusté afin de préparer la décision de soin. La formulation et la diffusion d'un tel modèle, pour idéal qu'il soit, n'en restent pas moins un acquis important des années 90 .

- la construction d'une cause générale: d'un autre côté les associations d'usagers de la santé ont manifesté leur capacité à élargir leurs points de vue particularistes et à effectuer des « montées en généralité » sur des problèmes globaux de politique de santé et de droits fondamentaux (en particulier la non discrimination et le bénéfice de la solidarité économique). Leurs actions conjuguées ont démontré que l'observation attentive des problèmes de prise en charge de maladies spécifiques (diabète, cancer, myopathies, mucoviscidose, $\mathrm{VIH}$ ) ou de handicaps (physique ou mentaux), et que les informations recueillies à l'occasion des soutiens psychologiques et sociaux apportés aux personnes atteintes étaient capables de produire une analyse élargie des problèmes de santé. Contrairement au lieu commun qui veut que chaque groupe soit enfermé dans le particularisme de ses besoins et l'égoïsme de ses revendications, ces associations ont démontré qu'elles partageaient de nombreux problèmes (qualité de l'information donnée au patient, sécurité hospitalière, conditions d'accès au dossier médical, choix des décisions thérapeutiques, nécessité d'un soutien psychosocial, assurabilité des risques aggravés de santé, etc.) et que seule une action collective soutenue était susceptible de mener à bien un vrai travail transversal aux différentes pathologies qu'elles représentaient. Habituellement on disqualifie les personnes et les groupes directement concernés de leur capacité à représenter par manque de détachement (leurs intérêts biaiseraient leur regard). On peut à partir de cet exemple soutenir l'analyse inverse : c'est en effet parce que ces groupes et leurs représentants sont très attachés à des causes spécifiques qu'ils ont pu agir de façon originale, puis mettre en relation leurs spécificités pour les agréger en une cause générale.

On retrouve ici tous les éléments de débat sur la question de la représentation et de la capacité à représenter. Les associations comme tous les mouvements collectifs tirent leur légitimité démocratique de leur force de mobilisation pour la défense de parties délaissées de l'intérêt général. Telle était le cas initialement des syndicats, des associations socio-éducatives et plus récemment des mouvements de consommateurs. L'élargissement de la cause défendue par les associations d'usagers est significative de l'ouverture progressive à la défense des intérêts de tous les citoyens. II faut ici rappeler le rôle tenu par les associations de consommateurs qui furent les premières à poser des enjeux généraux dès les années 70 (colorants alimentaires, veau 
aux hormones) et surtout dans les années 90 (affichage des honoraires, prix des médicaments, organisation de la médecine urgence, maisons de retraite). Et cela alors que l'attention des associations de personnes malades restait très liée à la prise en charge de telle ou telle pathologie (sida, diabète, cancer, etc.). C'est à partir de $1996^{1}$ que leur champ d'intérêt s'étendit à l'ensemble des questions hospitalières (information, dossier médical, accueil aux urgences, maladies nosocomiales). Mais le véritable élargissement a lieu entre 1998 et 2002 où c'est l'ensemble du système de soin qui fait l'objet d'examens et de revendications portant sur les enjeux de qualité et de meilleure organisation des prises en charge publiques ou privées. Enfin, après la loi du 4 mars 2002, ce sont les enjeux de financement et donc d'amélioration et de survie du système de santé qui vont entrer dans la cause collective portée par les usagers de soin et qui aboutira à leur entrée dans " la nouvelle gouvernance » de l'assurance maladie (CPAM et CNAM).

\section{Instrumentalisation de l'usager et inorganisation de la représentation}

Deux autres séries d'observation viennent cependant contrebalancer immédiatement les deux précédentes. Elles montrent que la participation est réduite bien souvent aux formes limitées du niveau un "managérial ", ou deux "modernisation". Les résistances du milieu professionnel sont ici renforcées par les lacunes des organisations d'usagers. Plusieurs observateurs ont déjà relevé les limites de l'objectif de coopération².

Instrumentalisation - II y a instrumentation lorsque l'invocation de la participation sert de couverture à la poursuite de pratiques inchangées. Les exemples sont nombreux de situations dans lesquelles le recours à des représentants d'usagers est réduit à une participation formelle. On peut en donner plusieurs formes:

- la participation " managériale » est un faux-semblant, c'est-à-dire qu'elle est conçue selon une approche de "bon management " d'une organisation basée sur des principes d'efficacité et de modernité. Le "cliento-centrisme » trouve ici une signification précise lorsque parmi les critères de "bon management " ou mieux d'« une gouvernance renouvelée », il est indispensable d'afficher une ouverture aux destinataires de l'action. C'est alors selon une stricte logique interne de modernisation à peu de frais qu'un représentant est associé à une instance ou une commission dont il ignore tout, dont les objectifs et méthode de travail ne lui seront jamais expliqués et dont on n'attend à peu près rien hormis sa présence. Le but principal est que sur le papier les responsables puissent afficher la nomination d'un représentant des usagers indépendamment de tout projet substantiel. Ainsi le " président du CISS " a été nommé membre du Conseil de surveillance de la CNAM en 2002 (avant la réforme), alors que le CISS n'a jamais eu de

\footnotetext{
${ }^{1}$ Entrée de deux représentants d'usagers dans les CA des hôpitaux, création de structures de conciliation pour les petits différents et les litiges, participation des représentants d'usagers à l'ANAES.

2 Gwénaelle Maudet, "La démocratie sanitaire : penser et construire et l'usager ", Lien social et politique, RIAC, n46, 2002, pp. 95-102 - Ivan Sainsolieu, L'hôpital et ses acteurs, Belin, 2003, pp. 122-125 - Frédéric Pierru, Hippocrate malade de ses réformes, Editions du Croquant, 2007, pp. 271-277.
} 
président avant octobre 2004 et que ce collectif n'avait pas encore affiché la moindre compétence sur les sujets de l'assurance maladie. Autre exemple, la direction de l'hospitalisation (du Ministère de la santé) a décidé de porter de deux à trois les représentants des usagers dans les conseils d'administration des hôpitaux. Il s'agit d'une décision unilatérale (le « plan hôpital 2007 » initial prévoyait, à l'inverse, une disparition de cette participation) qui vise à maintenir un équilibre entre collèges des conseils d'administration. Cette mesure n'a pas été concertée avec les associations et elle ne prend pas en compte la difficulté de motiver et former soudainement plus d'un millier de représentants supplémentaires ... ${ }^{1}$;

- la participation peut aussi se réduire à une fausse modernisation lorsque on se refuse à considérer qu'il s'agit d'une dynamique de coopération qui nécessite un certain nombre d'investissements en moyens et en temps. Parmi les nombreux exemples on peut en citer deux. Tout d'abord, la qualité d'une coopération repose sur la qualité des désignations de représentants, c'est pourquoi les associations ont été demandeuses, dès la préparation du projet de loi de 2002, d'une procédure d'agrément en matière de santé, afin d'éviter les représentants de façade ou d'opportunité. Passons sur les péripéties, il a fallu près de quatre ans de pressions soutenues pour que le ministère de la santé et la DGS mettent en place cette structure, la Commission nationale n'a été mise en place qu'en janvier $2006^{2}$. Alors que dans les autres ministères (consommation, jeunesse et sport, environnement) l'agrément est une procédure simple gérée par un bureau et un petit comité de sélection, ici on a mis la barre très haut comme si l'enjeu présentait une sensibilité particulière (deux élus nationaux, des représentants du Conseil d'Etat, de la Cour de cassation, etc.). De plus la commission prononce un avis qui lie le ministre ... ce qui est rarissime. Ensuite, la participation suppose des représentants qualifiés. L'UNAF qui dispose de plusieurs centaines de représentants dans des CA hospitaliers a réalisé une enquête instructive sur la façon dont ceux-ci ont été accueillis et intégrés dans ces structures. Seuls un tiers d'entre eux ont bénéficié d'une information et d'une présentation de l'établissement susceptible de leur permettre de concevoir leur rôle de façon active et pas seulement comme une présence passive autour d'une table. De plus, très peu sont associés à des activités en dehors du CA (commissions d'appel d'offre, de la qualité, procédure d'accréditation) ${ }^{3}$.

Les résultats des activités participatives conduisent aussi à regarder avec prudence les changements. Ainsi la question de l'information médicale du patient est un enjeu capital, mais elle est encore souvent conçue comme une action destinée à obtenir un consentement formalisé et non comme une activité destinée à permettre à une personne d'effectuer un choix. De plus, elle est fréquemment convertie en un système de protection des professionnels contre ce qu'ils estiment être les risques de contentieux. Si l'on regarde maintenant les résultats de la participation des usagers aux groupes

\footnotetext{
${ }^{1}$ Décret du 7 juillet 2005 sur les Conseils d'administration, commissions médicales, etc. des établissements de santé.

2 M. Mattei se refusait à mettre en œuvre cette partie de la loi de 2002. Les raisons sont restées floues mais on comprenait bien que la crainte de devoir refuser un agrément à des associations locales faisant dans le caritatif hospitalier et animées par des membres de l'élite notabilière (et souvent leur épouse ...) était un frein important.

${ }^{3}$ UNAF, Bloc-Note santé, $n^{\circ} 54$, octobre 2002.
} 
de travail de l'ex-ANAES (aujourd'hui Haute autorité de santé) sur l'accréditation (certification) des établissements, on constate à quel point il est toujours difficile de faire introduire des indicateurs de résultats (délais d'attente, évolution des contaminations nosocomiales, suivi précis des réhospitalisations, etc.), les professionnels préférant le plus souvent en rester à des indicateurs de procédure et des évaluations de conformité à ces procédures.

\section{Le manque d'organisation de la représentation}

La participation reste aussi souvent vide de sens en raison de la faiblesse du contrôle de la représentation elle-même. Si l'objectif des mobilisations associatives était de faire des représentants des usagers de véritables " acteurs " du système de santé, cette ambition se heurte en pratique à différentes limites dont les principales sont les suivantes. Tout d'abord, le représentant tire sa légitimité d'un mandat, cela exige de lui qu'il rende des comptes à ses mandants. Le but n'est pas d'occuper telle ou telle place, ni même un maximum de place, mais de donner un sens à cette activité. C'est à dire de veiller à être un porte-parole responsable, capable de collecter des informations, de rechercher les besoins à satisfaire, de faire le lien entre des cas particuliers et des situations générales. Hors les associations veillent peu, en général, à maintenir des liens étroits avec leurs représentants afin de faire le point sur les mandats et de faire évoluer ceux-ci. Cette préoccupation est d'autant plus importante que le représentant est souvent seul face à des groupes de professionnels ou de spécialistes. En tant que profane il est structurellement mal équipé pour faire entendre une parole différente et construire une position autonome de celles qui prévalent dans ces instances. II a donc un besoin particulier d'être soutenu et formé, de participer à des débats avec ses pairs, et d'être nourri de propositions. On retrouve ici la question classique en sociologie des mouvements sociaux de la double coupure: d'une part, celle qui s'observe fréquemment entre une élite associative spécialisée en pratique dans la participation et les militants de base ; et d'autre part celle qui existe toujours entre les militants et les populations au nom desquelles ils agissent ${ }^{1}$.

Ensuite, les représentants (et par voie de conséquence, les associations qui les mandatent) peuvent facilement céder à la facilité de gérer les situations dans lesquelles ils sont amenés à participer, d'intégrer les contraintes (limites de moyens, tensions inter-professionnelles), de composer avec des décisions qui leur échappent (ordre du jour des CA) ${ }^{2}$. La volonté de coopérer se traduit souvent par une perte de vigilance et une moindre capacité à formuler des revendications. La représentation est toujours menacée par une tactique commode dite du "pull sur la chaise », qui est une façon d'être présent sans s'engager. La formulation et la défense active de positions collectives sont

\footnotetext{
1 Frédéric Pierru, op. cit.

2 Beaucoup sont purement gestionnaires, on parle de budgets, de personnels, rarement d'amélioration de l'organisation ou d'objectifs de qualité. Obtenir une fois l'an l'inscription d'un point sur le bilan des plaintes et des conciliations, ou obtenir l'élargissement des groupes d'évaluation de la qualité à des non professionnels est un vrai challenge.
} 
ou fiction utile?

pourtant partie intégrante de la représentation dans la mesure où celle-ci a la charge de l'expression au nom de tous ceux qui ne le peuvent pas. Mais pour tenir cette mission de façon constructive, encore faut-il que les représentants soient motivés à prendre des initiatives, à collecter des informations, à échanger avec des interlocuteurs diversifiés, à identifier des besoins et les traduire en propositions. C'est une activité qui ne peut pas relever des seules initiatives individuelles. Mais peu d'associations sont réellement conscientes de ces problèmes; trop heureuses en général de trouver des représentants bénévoles, elles les laissent souvent dépourvus d'accompagnement et de mandats régulièrement validés.

Ainsi les résistances institutionnelles qui incitent à l'instrumentalisation de la participation et le manque de vigilance des associations qui se satisfont d'une représentation relativement inorganisée, tous ces facteurs expliquent pourquoi la participation dépasse rarement le niveau deux d'une action de modernisation qui se satisfait d'activités formelles et de communication et ne se donne pas les moyens de construire une coopération apte à induire des changements substantiels.

\section{L'Usager, une fiction nécessaire?}

Ni administré discipliné, ni client opérant des choix économiques rationnels l'usager est une figure intermédiaire porteuse de nombreuses ambiguïtés. Les mobilisations collectives qui ont eu lieu depuis vingt ans dans le domaine de la santé, ainsi que l'importance des reconnaissances légales et institutionnelles qui en ont découlé ne doivent pas faire illusion. L'usager n'est pas plus " au centre du système de soin » que le "client n'est roi », sauf à prendre le discours managérial à la lettre. D'un côté, la dissymétrie des positions entre professionnels de la santé et usagers a été réduite, en particulier par un accès massif à l'information médicale (via les associations relayées aujourd'hui par internet) et par la reconnaissance de droits et la mise en place de procédures de règlement des conflits. Mais d'un autre côté, le poids récurrent des approches techniciennes déréalisantes et les contraintes budgétaires n'incitent guère les professionnels à s'engager dans des démarches coopératives approfondies. Celles-ci ont du mal à se généraliser car les acteurs associatifs peinent à bien investir les opportunités qui sont offertes aux usagers du système de soin. Certes l'empowerment est un horizon qui pourrait permettre d'avancer vers une démocratie participative. Mais les observations empiriques montrent qu'il est rare que l'ensemble des acteurs concernés (usagers inclus) parvienne à dépasser les formes de gestion managériale ou de modernisation formelle. Dans ces deux cas, les changements sont de pure surface et maintiennent une profonde dissymétrie de position. Le scénario du pire verrait les personnes " objet de soin " céder la place à un "usager potiche" cautionnant de sa présence muette des décisions toujours plus complexes et extérieures à lui. Si différents éléments permettent de considérer qu'une dynamique positive est amorcée, d'autres conduisent à tempérer sérieusement ce point de vue. En effet, une énorme ambiguïté pèse toujours sur cet usager qui est davantage celui au nom de qui on parle que celui qui s'exprime directement. Professionnels et associatifs sont autant concernés l'un que l'autre par cette interrogation. 
Dans un tel système de participation confuse et marquée de tant d'incertitudes, l'usager occupe une place extrêmement floue. Le rôle qu'il tient est en grande partie virtuel et se trouve au confluent de trois regards contraires: celui des institutions de santé, celui de tous les usagers silencieux, celui enfin des quelques usagers revendicatifs. Dans ce sens le risque du " cliento-centrisme " serait un accord implicite entre l'ensemble des acteurs concernés sur une référence abstraite en forme de standard de qualité de service minimale, garantie par des procédures et mesurée par quelques indicateurs. On irait alors aux antipodes de l'ambition de certains associatifs comme Daniel Defert (créateur de l'association Aides ${ }^{1}$ ) qui souhaitait faire de la personne malade un réformateur social, c'est-à-dire capable d'interroger et de faire évoluer non seulement les pratiques médicales, mais plus largement les mentalités et les rapports sociaux par la confrontation à une expérience structurée de la souffrance et de la mort.

"Daniel Defert, "Du deuil à la lutte", Eric Favereau, Nos années Sida 25 ans de guerres intimes, La Découverte, 2005, pp. 65-76. 